\title{
Synergistic Neuroprotective Effects of Two Herbal Ingredients via CREB-Dependent Pathway
}

\section{OPEN ACCESS}

Edited by:

Marianthi Papakosta,

Pfizer, USA

Reviewed by:

Erik B. Oleson,

University of Colorado Denver, USA

Stephen Morairty,

SRI International, USA

*Correspondence:

Yuan $\mathrm{Hu}$

huyuan1980619@sina.com

Ping Liu

liupingpla@126.com

tThese authors have contributed equally to this work.

Specialty section:

This article was submitted to

Neuropharmacology,

a section of the journal

Frontiers in Pharmacology

Received: 02 June 2016 Accepted: 12 September 2016 Published: 27 September 2016

Citation:

Liu $X$, Wang $D$, Zhao $R$, Dong $X$,

Hu Y and Liu P (2016) Synergistic Neuroprotective Effects of Two Herbal Ingredients via CREB-Dependent

Pathway. Front. Pharmacol. 7:337. doi: 10.3389/fphar.2016.00337

\author{
Xu Liư', Dongxiao Wangt, Runqing Zhao, Xianzhe Dong, Yuan Hu* and Ping Liu* \\ Department of Clinical Pharmacology and Pharmacy, Pharmacy Care Center, Chinese PLA General Hospital, Beijing, China
}

As two natural oligosaccharide esters, 3,6'-Disinapoyl sucrose (DISS) and tenuifolisideA (TFSA) are originating from the root of Polygala tenuifolia Willd, a traditional Chinese medicine used in treatment of mental disorders. Previous reports have shown that both of them possess in vitro neuroprotective effects by stimulating different upstream pathways related with cyclic AMP-responsive element-binding protein (CREB). In the present study, we investigated the additive neuroprotective effects of DISS and TFSA on Glu-induced damage of SY5Y cells and purposed the possible underlying mechanism. The interaction between DISS and TFSA showed a clear-cut synergistic effect as evidenced by combination index $(\mathrm{Cl})$. Additional evidence from biochemical (NOS activity) assays confirmed their additive inhibition on the Glu-induced NOS hyperactivation. Moreover, we showed that co-treatment of DISS and TFSA resulted in an additively up-regulated phosphorylation of CREB as well as increased expressions of CRTC1 and BDNF. Neuroprotective effects of DISS and TFSA on Glu-induced decrease in cell viability were blocked by MAPK/ERK1/2 inhibitor (U0126) and PI3-K inhibitor (LY290042). Nevertheless, the CRTC1 or BDNF expression induced by these two compounds was significantly reduced in the presence of either ERK or PI3-K inhibitor, indicating that the two oligosaccharide esters shared some common pathways in the regulation of CREB-BDNF pathway. Taken together, we, for the first time, showed that DISS and TFSA exerted the additive neuroprotective effects on CREB-BDNF signaling pathway through complementary mechanisms.

Keywords: DISS, TFSA, CREB, synergistic effect, neuroprotective effect

\section{INTRODUCTION}

As a common mental disorder, depression is estimated to affect 350 million people in the world by 2030 (Mathers et al., 2008). Conventional medicines for many depressed patients address only low efficacy but with untoward side effects (Blumenthal et al., 2012). Chinese herbs have a very long history for the use in the treatment of depression with few side effects reported. Extract of Radix Polygalae (the root of Polygala tenuifolia Willd) has been used as an antidepressant, a tranquilizer and an antipsychotic agent in the past several years (Chang and But, 1986; Hu et al., 2009; Liu et al., 2010). 3,6'-Disinapoyl sucrose (DISS) and tenuifoliside A (TFSA) are the principle active ingredients in the extract of Radix Polygalae. Our previous studies have indicated that both of DISS and TFSA have neuroprotective effects in different in vitro models (Hu et al., 2010, 2011, 2012). In addition, both can stimulate the expression and phosphorylation of key proteins in the 
cAMP response element-binding protein (CREB) signaling pathway (Liu et al., 2010; Dong et al., 2014).

cAMP response element-binding protein with its upstream and downstream effectors is thought to play a role in the pathophysiology of depression as well as in efficacy of antidepressant treatment (Majumder et al., 2004; Nair and Vaidya, 2006; Sakamoto et al., 2011). A serial of activityinducible kinases (such as protein kinase $\mathrm{C}$, Ca2+/CaMdependent kinases II and IV, protein kinase A, ribosomal S6 kinase, AKT, mitogen/stress-activated kinase, and MAPK kinase 2) can increase the phosphorylation of CREB (Ghosh et al., 1994; Ginty et al., 1994; Tan et al., 1996; Hardingham et al., 1999; Li et al., 2011). Meanwhile, CREB-regulated transcription co-activators (CRTCs) can also dramatically increase CREB-mediated transcriptional activity independently of the phosphorylation status of CREB (Reichardt, 2006). Both of the above-mentioned mechanisms upregulate the downstream neurotrophic factor (BDNF) and affect neuronal proliferation, synaptic function, and synaptic plasticity.

Synergy may occur when two or more herbal ingredients are combined, which can mutually enhance each other's effect when compared with administration alone (Ma et al., 2009). Therefore, we combined DISS and TFSA to determine if such a combination produced an additive or synergistic neuroprotective effect.

In the present study, we specifically aimed to assess the combination effect of two compounds, DISS and TSFA, in mediating neuroprotective effects in vitro and to further explore their additive mechanism via CREB pathway [CRTC1, pCREB (phosphor-CREB), BDNF]. Our goal was to investigate the potential usefulness of combining candidate active oligosaccharide esters for the treatment of mental disorders, by which we could reduce the risk of adverse effects by lowering their concentrations.

\section{MATERIALS AND METHODS}

\section{Materials}

3,6'-Disinapoyl sucrose and TSFA were isolated from Radix Polygalae, identified by a combination of spectral methods (UV, IR, MS, and NMR) and purified by HPLC (purity >90\%). U0126 and LY294002 were purchased from Selleck Chemicals LLC (Houston, TX, USA). Dulbecco's modified Eagle's medium (nutrient mixture F-12; DMEM/F-12) was obtained from Gibco (Carlsbad, CA, USA). Fetal bovine serum (FBS) was supplied by Hyclone (Logan, UT, USA). Polyclonal rabbit antibodies against BDNF, CREB, pCREB, CRTC1, and $\beta$-actin were all provided by Bioworld Technology (Atlanta, GA, USA) or Abcam (Burlingame, CA, USA).

\section{Cell Culture}

SH-SY5Y human neuroblastoma cells were obtained from the American Type Culture Collection (Rockville, MD, USA) and cultured in DMEM/F-12 supplemented with $10 \%$ heatinactivated FBS and $1 \%$ penicillin-steptomycin $(50 \mathrm{U} / \mathrm{mL})$. The cells were maintained in a humidified atmosphere with $5 \% \mathrm{CO}_{2}$ at $37^{\circ} \mathrm{C}$. The culture medium was refreshed every other day.

\section{Cell Viability Assay and Synergy Experiments}

Cells were seeded into 96-well plates at a density of approximately $2 \times 10^{3}$ cells/well and then grown for $24 \mathrm{~h}$. Subsequently, cells were cultured with serum-free medium $(0.1 \%$ BSA in growth medium without FBS) for another $24 \mathrm{~h}$ and then treated with DISS or TFSA at desired concentrations (15.6252,000 $\mu \mathrm{M})$. After 48 -h treatment, cell viability was determined by Cell Counting Kit-8 (CCK-8; Keygen Biotech., Nanjing, China). Results were from six independent experiments, and each experiment was run in triplicate. The data were presented as percentage relative to untreated cells. The drug response curve was generated based on the determined relative cell viability, and $50 \%$ effective concentration $\left(\mathrm{EC}_{50}\right)$ was measured using software Graphpad Prism5 (Roscilli et al., 2016). EC 50 of combination was tested on TFSA at a fixed concentration $(40 \mu \mathrm{M})$ plus DISS at various concentrations $(600,300,150,75,37.5,18.75 \mu \mathrm{mol} / \mathrm{L})$. Combination effects of DISS and TFSA treatment were evaluated by calculating the combination index (CI) value as previously reported by Chou (Chou, 2006). $\mathrm{CI}=\mathrm{EC}_{50 \text { (DISS plus TFSA) }} / \mathrm{EC}_{50}$ (DISS alone) $+\mathrm{C}_{\mathrm{TFSA}} / \mathrm{EC}_{50}$ (TFSA alone). $\mathrm{C}_{\mathrm{TFSA}}$ was the TFSA concentration used in combination. In this analysis, synergism was defined as follows: $\mathrm{CI}>1.1$, antagonism; $1.1<\mathrm{CI}<0.9$, additive effect; $0.9<\mathrm{CI}<0.3$, synergic effect; and $\mathrm{CI}<0.3$, strong synergism.

\section{Inhibition Experiments}

Briefly, cells were seeded into a 96-well plate or 6-well plate at a density of approximately $3 \times 10^{3}$ cells/well or $1 \times 10^{4}$ cells/well with full growth medium. After $24 \mathrm{~h}$, medium was refreshed by serum-free medium and incubated $24 \mathrm{~h}$. Cells were exposed to $8 \mathrm{mM}$ glutamate (Glu) for $24 \mathrm{~h}$, followed by treatment with DISS $(75 / 150 \mu \mathrm{M})$, TFSA $(25 / 50 \mu \mathrm{M})$ alone or combinations of DISS and TFSA at desired concentrations with or without antagonists (U0126/LY294002) for 24 or 48 h (assay BNDF mRNA, western blot), and each concentration of the inhibitor was established according to a previous study with minor modifications ( $\mathrm{Hu}$ et al., 2014).

\section{Measurement of NOS and iNOS Activities}

Cells were seeded into 6-well plates at a density of approximately $1 \times 10^{4}$ cells/well cells and grown for $24 \mathrm{~h}$. Subsequently, cells were exposed to $8 \mathrm{mM}$ Glu for another $24 \mathrm{~h}$. After different treatments for $48 \mathrm{~h}$, cells were collected and re-suspended in PBS. Cell suspension was centrifuged, and the supernatant was collected into EP tubes and then stored at low temperature $\left(0^{\circ} \mathrm{C}\right)$. NOS and iNOS activities as well as NO content were determined using a reagent kit (Nanjing Jiancheng Bioengineering Institute, Nanjing, China) using an ultraviolet spectrophotometer at a wavelength of $540 \mathrm{~nm}$.

\section{BDNF Expression at the mRNA Level}

Total RNA was extracted from cells using Trizol reagent. RNA concentration was determined using an Eppendorf BioPhotometer plus (Eppendorf, Germany). Subsequently, purified RNA was reversely transcribed into cDNA using iScript 
cDNA Synthesis Kit (Bio-Rad, Hercules, CA, USA) according to the manufacture's instructions. BDNF expression at the mRNA level was assessed by qPCR using SsO Fast Eva Green Supermix (Bio-Rad, Hercules, CA, USA) according to the manufacturer's instructions. GAPDH was selected as the housekeeping gene. The following sequences were used in the study:

\section{BDNF up Primer: GACGGTCACAGTCCTAGAGAA,}

BDNF low primer CCTTATGAATCGCCAGCCAAT;

GAPDH up primer ACTTCAACAGAGACACCCACTC,

GAPDH low primer TCTCTCTTCCTCTTGTGCTCTTGC.

Briefly, after an initial denaturation step at $95^{\circ} \mathrm{C}$ for $30 \mathrm{sec}$, amplifications were carried out with 40 cycle at a melting temperature of $95^{\circ} \mathrm{C}$ for $5 \mathrm{sec}$, an annealing temperature of $60^{\circ} \mathrm{C}$ for $5 \mathrm{sec}$, and an extension temperature of $65^{\circ} \mathrm{C}$ for $5 \mathrm{sec}$. Standard curves were generated for each gene, and the gene expression at the mRNA level was calculated relative to serial dilution of cDNA as described in Bio-Rad iQ5 System (California, USA). All experiments were conducted in triplicates. Relative expression was estimated using the $2^{-\Delta \Delta \mathrm{Ct}}$ method.

\section{Western Blotting}

After 48-h treatment, cells were collected and washed twice with ice-cold PBS. Subsequently, cell pellet was lyzed in lysis buffer (1\% Triton, $20 \mathrm{mM}$ Tris- $\mathrm{HCl}, 150 \mathrm{mM} \mathrm{NaCl}, 1 \mathrm{mM}$ EDTA, pH 7.5) supplemented with one tablet of complete protease inhibitor cocktail (Roche, Laval, QC, Canada) and phosphatase inhibitor cocktail (Sigma, St. Louis, MO, USA), and the cell lysates were centrifuged at $1,000 \mathrm{~g}$ for $10 \mathrm{~min}$ at $4^{\circ} \mathrm{C}$. Total amounts of proteins in each sample were determined by BCA kit, and the protein concentrations of all samples were adjusted to be the same. Supernatants containing $20 \mu \mathrm{g}$ proteins were separated on 10\% SDS-PAGE and electrotransferred onto polyvinylidenedifluoride (PVDF) membranes. Blots were

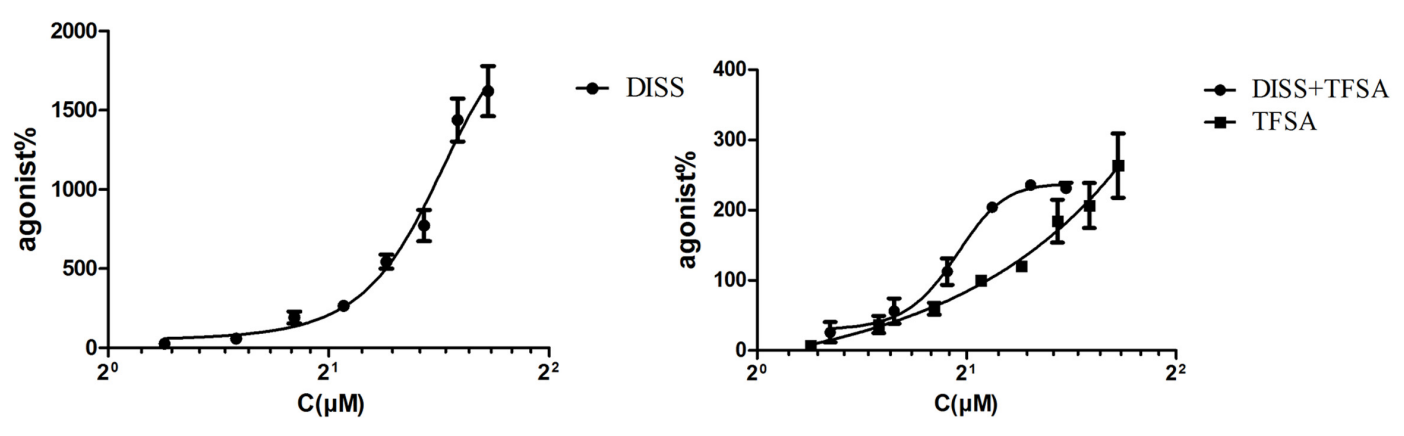

FIGURE 1 | EC $_{50}$ of combination of DISS (3,6'-Disinapoyl sucrose) and TFSA (tenuifolisideA). Cells were treated with DISS or TFSA at desired concentrations $(15.625-2,000 \mu \mathrm{M})$ then cell viability were determined by cell counting kit-8. DISS or TFSA response curve was generated based on the determined relative cell viability, $\mathrm{EC}_{50}$ was measured. $\mathrm{EC}_{50}$ of combination was tested on TFSA at a fixed concentration ( $\left.40 \mu \mathrm{M}\right)$ plus $\mathrm{DISS}$ at various concentrations $(600,300$, $150,75,37.5,18.75 \mu \mathrm{mol} / \mathrm{L})$. The $\mathrm{EC}_{50}$ of DISS, TFSA and the combination of DISS and TFSA was $606.4 \pm 23.3 \mu \mathrm{M}, 237.8 \pm 13.3 \mu \mathrm{M}$, and $83.86 \pm 1.06 \mu \mathrm{M}$, respectively. $\mathrm{Cl}$ value of combination was $0.31(\mathrm{Cl}<1$ means synergic effect).

A

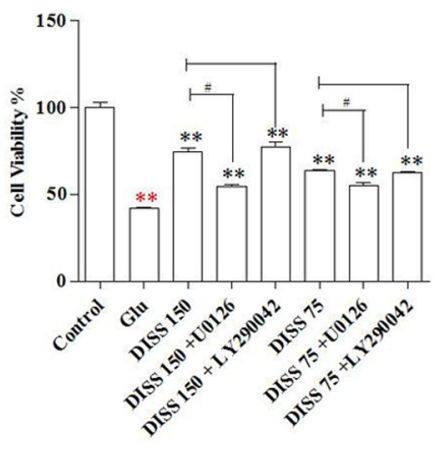

B

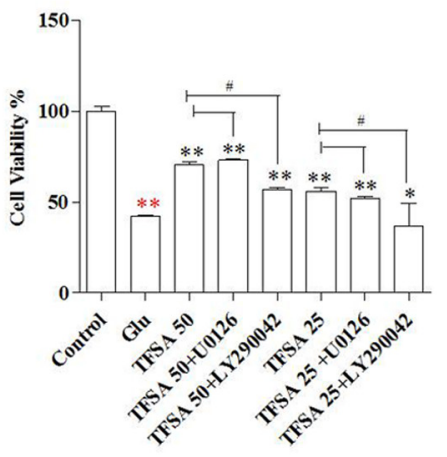

C

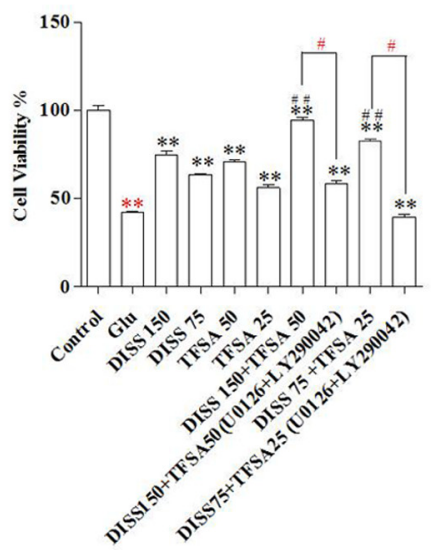

FIGURE 2 | Combination effect of DISS and TFSA on cell viability in SY5Y cells $(\boldsymbol{n}=\mathbf{1 8})$. SY5Y cells were exposed to 8 mM Glu for 24 h, followed by combination treatment of DISS and TFSA as well as inhibitors (U0126, LY294002). (A) Cells were treated by DISS and inhibitors. (B) Cells were treated by TFSA and inhibitors. (C) Cells were treated by combination of DISS, TFSA and inhibitors. ${ }^{*} p<0.05$ and ${ }^{* *} p<0.01$ compared with the Glu group. Red ${ }^{* *} p<0.01$ compared with control group. ${ }^{*} p<0.05,{ }^{*} p<0.001$, and red ${ }^{\#} p<0.05$ compared with its compound alone. 
blocked in Tris buffered saline containing $0.1 \%$ Tween-20 (TBST) and $5 \%$ non-fat-dried milk at room temperature for $1 \mathrm{~h}$. Next, the membranes were incubated with the respective primary antibodies (anti-CREB 1:500, anti-phospho-CREB 1:500, antiBDNF 1:2,000, and CRTC1 1:10,000) overnight at $4^{\circ} \mathrm{C}$. Following three washes with TBST, the blots were incubated with the secondary horseradish peroxidase-conjugated antibody $(1: 6,000)$ at room temperature for $1 \mathrm{~h}$, and optical density of protein bands was determined using an image analysis system (Bio-Imaging Analyzer, UVP). All the results of western blot consisted of three independent experiments, and each independent experiment was performed in triplicate.

\section{Statistical Analysis}

Data were presented as mean $\pm \mathrm{SD}$. Comparisons were carried out using one way analysis of variance (ANOVA) followed by Tukey-Kramer's test for post hoc analysis. All statistical analyses were performed using ANOVA by the PRISM software (GraphPad software, San Diego, CA, USA). $P<0.05$ was considered as statistically significant.

\section{RESULTS}

\section{Combination of DISS and TFSA Inhibits Glu-Induced Decrease in Cell Viability}

Figure 1 shows that the $\mathrm{EC}_{50}$ values of DISS and TFSA were $606.4 \pm 23.3 \mu \mathrm{M}$ and $237.8 \pm 13.3 \mu \mathrm{M}$, respectively. The fixed concentration of $40 \mu \mathrm{M}$ TFSA in combination was according to $\mathrm{EC}_{20}$ of TFSA $(40.71 \pm 2.47 \mu \mathrm{M})$. The $\mathrm{EC}_{50}$ of combination of DISS and TFSA was $83.86 \pm 1.06 \mu \mathrm{M}$. CI value of co-treatment
A
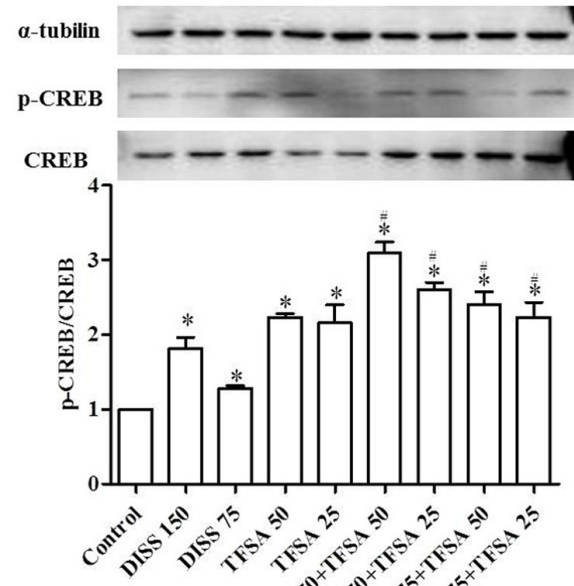

की को के की

C
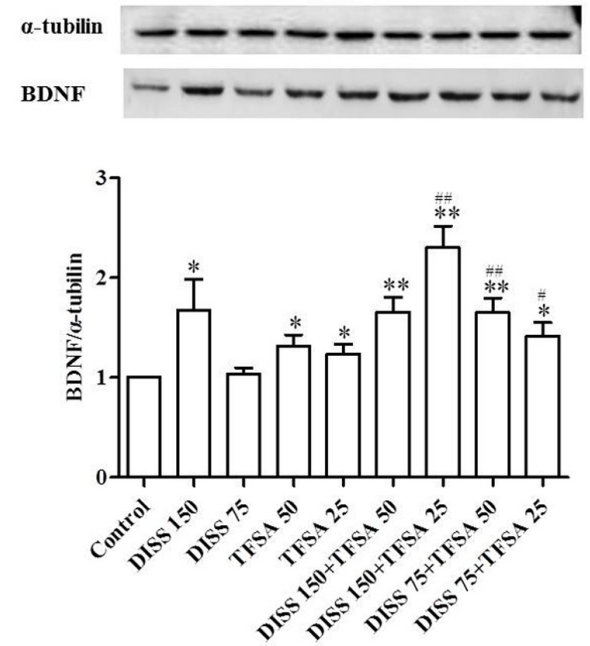
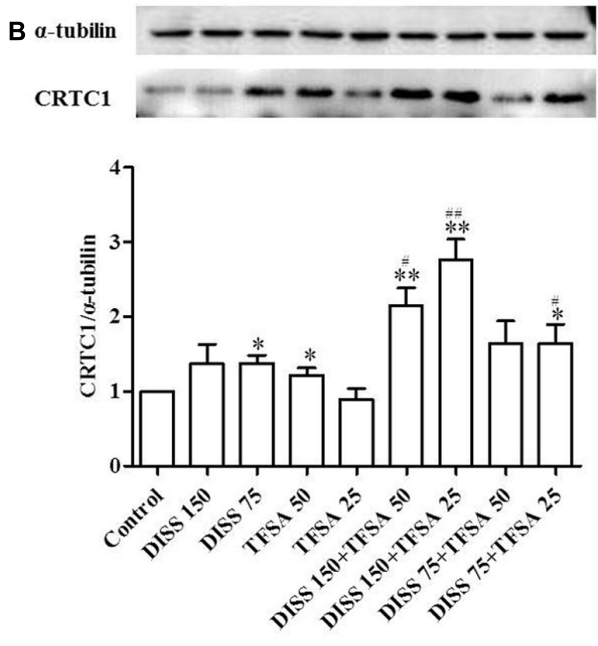

D

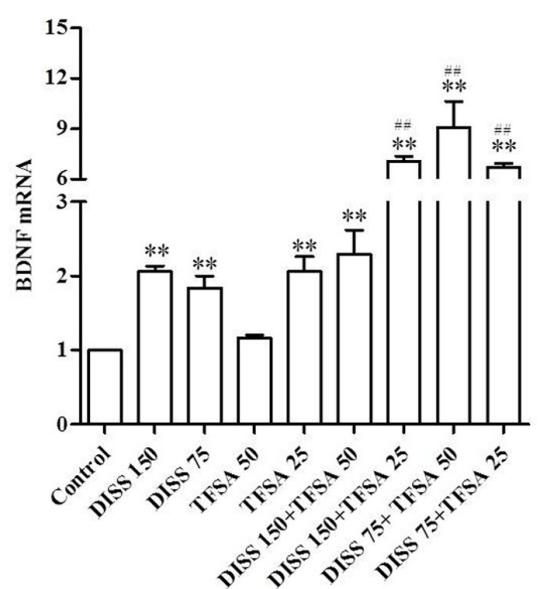

FIGURE 3 | Combination effect of DISS and TFSA on PCREB (phosphor-CREB)/CREB (cAMP response element-binding protein), BDNF, and CRTC1 expressions at the protein level as well as BDNF expression at the mRNA level in SY5Y cell $(\boldsymbol{n}=\mathbf{1 8})$. SY 5 Y cells were treated with $75 \mu \mathrm{M}$ or $150 \mu \mathrm{M}$ DISS, $25 \mu \mathrm{M}$ or $75 \mu \mathrm{M}$ TFSA, or combination of DISS and TFSA for $24 \mathrm{~h}$. (A,B,C) Combination effect of DISS and TFSA on pCREB/CREB, CRTC1, and BDNF expressions at the protein level. (D) Combination effect of DISS and TFSA on BDNF expression at the mRNA level. ${ }^{*} p<0.05$ and ${ }^{* *} p<0.01$ compared with the control group. ${ }^{\#} p<0.05$ and ${ }^{\# \#} p<0.01$ compared with its compound alone. 
was 0.31 , suggesting that combination of DISS and TFSA possessed a synergistic effect. Next, we investigated whether the combination of DISS and TFSA could have an additive or synergistic effect in preventing neuronal damage in SH-SY5Y cells. In order to lower the possible side effects of the compounds according to our previous experience, the concentrations of DISS and TFSA were set to $150 / 75 \mu \mathrm{M}$ and $75 / 50 \mu \mathrm{M}$, respectively. The $\mathrm{EC}_{20}$ of DISS alone was $214.2 \pm 1.23 \mu \mathrm{M}$. In combination, we chose 150 or $75 \mu \mathrm{M}$ DISS that lower than $\mathrm{EC}_{20}$ of DISS and increased slightly TFSA concentrations based on $\mathrm{EC}_{20}$ of TFSA alone.

\section{The Effects of MAPK/ERK1/2 and PI3-K/Akt Inhibitors on the Protective Effects of DISS and TFSA against Glu-Induced Decrease in Cell Viability and Proliferation Rate}

Based on this Glu-induced decrease in cell activity, the neuronal cytotoxicity of Glu could be partially inhibited by the pretreatment of DISS or TFSA in a dose-dependent manner. More importantly, the co-treatment of TFSA and DISS at two testing doses $(75 \mu \mathrm{M}+25 \mu \mathrm{M}$ and $150 \mu \mathrm{M}+25 \mu \mathrm{M})$ could produce a significant neuroprotective activity as compared with individual compounds alone, exhibiting increased cell viability from $48 \%$ to more than $85 \%$. Figures 2 A,B shows that such a protective effect of DISS at 150 and $75 \mu \mathrm{M}$ could be blocked by the MAPK/ERK1/2 inhibitor, U0126 (10 $\mu \mathrm{M})$, but not by the PI3-K inhibitor, LY290042 $(20 \mu \mathrm{M})$. In contrast, the LY290042, but not U0126, inhibited the neuroprotective effect of TFSA. Moreover, the inhibitory effect of combination of U0126 and LY290042 became even more obvious, showing reduced cell viability from $85 \%$ to less than $60 \%$ (Figure 2C).

\section{Combination Effects of DISS and TFSA on the Expression of CREB Pathway Related Key Targets}

In order to further understand the mechanism of the combination effect of DISS and TFSA on the neuroprotection, we investigated the expressions of CREB, CRTC1, pCREB, and BDNF. DISS or TFSA alone could induce the protein expression of pCREB/CREB at both doses, and additive effect was observed for the combination of DISS and TFSA (Figure 3A). In addition, TFSA at $25 \mu \mathrm{M}$ had no effect on the CRTC1 expression. However, combinations of $25 \mu \mathrm{M}$ TFSA and 150 or $75 \mu \mathrm{M}$ DISS both significantly increased the CRTC1 expression (Figure 3B). Meanwhile, the combinations of DISS and TFSA at different ratios all significantly enhanced the BDNF expression at the mRNA and protein levels (Figures 3C,D). Moreover, BDNF expression at the mRNA level was induced for more than threefold by combinations of $150 \mu \mathrm{M}$ DISS and $25 \mu \mathrm{M}$ TFSA; $75 \mu \mathrm{M}$ DISS and $50 \mu \mathrm{M}$ TFSA; or $75 \mu \mathrm{M}$ DISS and $25 \mu \mathrm{M}$ TFSA compared with compounds alone (Figure 3D).

\section{The Effects of ERK1/2 and PI-3K Inhibitors Are Involved in the Regulation of CRTC1 and BDNF Expressions by Induced by DISS and TFSA}

Considering the various CREB pathways activated by DISS and TFSA, we assessed the signaling mechanisms involved in CRTC1 and BDNF regulation using two inhibitors. Figure 4 reveals that treatment of DISS or TFSA increased the expression of BDNF and CRTC1, but such an increase could be partially attenuated by either U0126 or LY294002. PI3-K inhibitor LY294003 showed a similar but weaker inhibitory effect on DISS-induced CRTC1 expression compared with

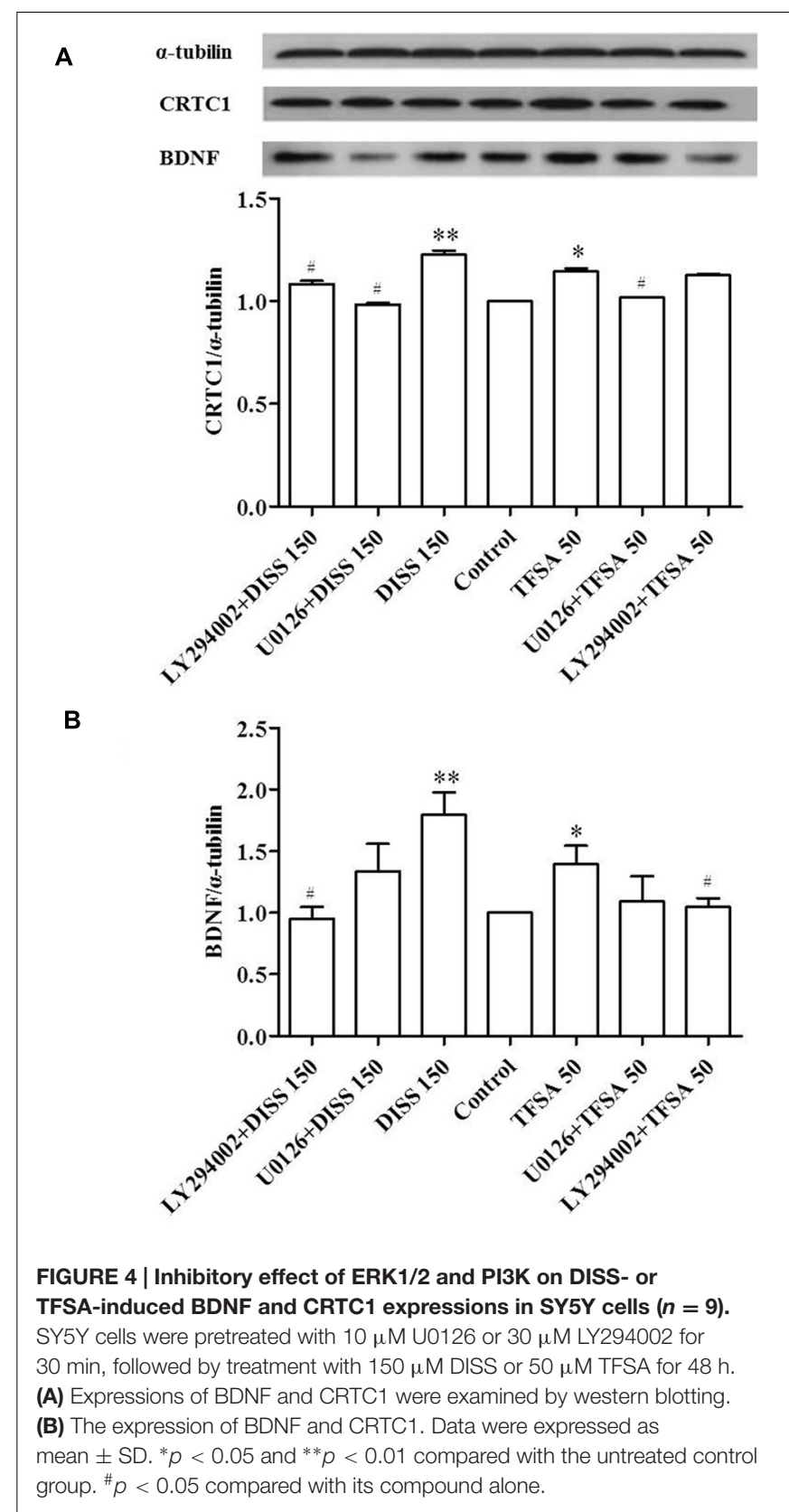




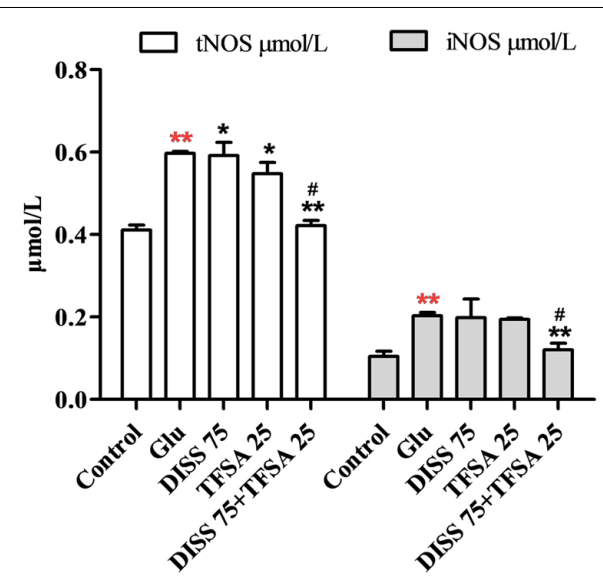

FIGURE 5 | Combination effect of DISS and TFSA on levels of tNOS and iNOS in SY5Y cells $(\boldsymbol{n}=\mathbf{1 8})$. SY5Y cells were exposed to Glu for $24 \mathrm{~h}$, after which Glu was changed to DISS and TFSA combination medium for $48 \mathrm{~h}$. Levels of tNOS and iNOS were determined by a reagent kit ** $p<0.01$ compared with Glu; ** $p<0.01$ compared with the control; * $p<0.05$, ${ }^{\#} p<0.01$ compared with its compound alone.

U0126 (Figure 4A). The LY294002 decreased the DISS- or TFSA-induced BDNF expression by 47 and 25\%, respectively. The ERK1/2 inhibitor U0126 decreased the DISS- or TFSAinduced BDNF expression by 25 and 21\%, respectively. (Figure 4B). A bigger decrease in DISS- or TFSA-induced BDNF expression was caused by LY294003 compared with U0126 (Figure 4B).

\section{Combination Effects of DISS and TFSA on tNOS and iNOS Levels}

To further investigate the additive neuroprotection of DISS and TFSA against the Glu-induced cell injury, we examined the levels of tNOS and iNOS, which play an important role in cell apoptosis or neurodegenerative disorders (Chen et al., 2016; Zhu et al., 2016). Figure 5 shows that Glu significantly increased the levels of tNOS and iNOS. DISS or TFSA treatment alone decreased the intracellular level of tNOS by $4-8 \%$, but showed no effect on iNOS level. However, co-treatment of DISS and TFSA significantly decreased such an elevation of tNOS and iNOS levels by 29.3 and $40.5 \%$, respectively. These results suggested that the combination of DISS and TFSA could more vigorously inhibit the Glu-induced NO overproduction.

\section{DISCUSSION}

The combination strategy offers a series of potential advantages, including less side effects and a more rapid or effective clinical response. In the current study, combination of the two active oligosaccharide esters demonstrated additive neuroprotective effects against Glu-induced damage. Compared with DISS or TFSA alone, their combination also significantly increased the expressions of pCREB, CRTC1, and BDNF.

Several studies have shown that DISS and TFSA play an antidepressant and neuroprotective role. DISS protects human neuroblastoma cells ( $\mathrm{SH}-\mathrm{SY} 5 \mathrm{Y}$ ) from Glu- or $\mathrm{H}_{2} \mathrm{O}_{2}$-induced damage, and DISS-mediated regulation of BDNF expression is associated with CREB-mediated transcription of BDNF and upstream activation of ERK1/2 and CaMKII (Hu et al., 2014). In our previous study, DISS possesses antidepressantlike properties, and its underlying mechanism might also be related to effects on hippocampal neuroplasticity gene BDNF (Hu et al., 2010). TFSA, with the similar chemical structure, can also promote the proliferation of C6 glioma cells, and its mechanism has been related to TrkB/BDNF/ERK and $\operatorname{TrkB} / \mathrm{BDNF} / \mathrm{PI} 3-\mathrm{K}$ signaling pathways (Dong et al., 2014).

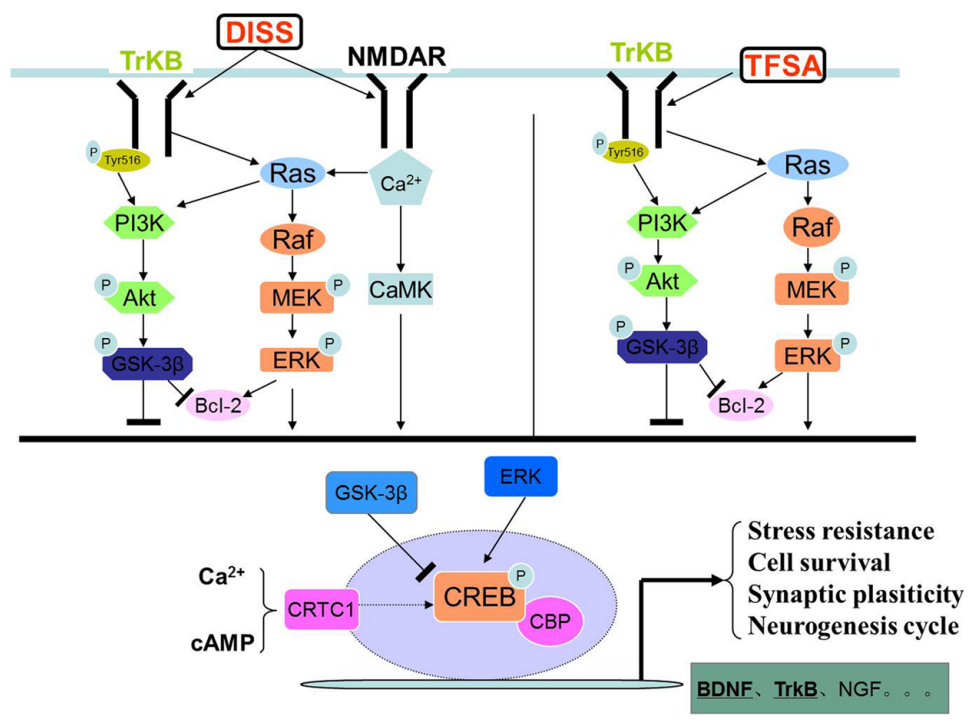

FIGURE 6 | The graph of DISS and TFSA synergistically activated the PI-3K/Akt and MAPK/ERK pathways. 
Over the past decades, studies have suggested that neurotrophins and related signaling cascades are involved in the pathophysiology of depression. CREB upregulation may activate downstream targets, such as BDNF, after antidepressant treatment. In fact, a series of elegant studies have also shown that PI-3K/Akt and MAPK/Erk pathways, which can promote neuronal growth and survival, are two key signaling cascades regulating CREB/BDNF activation (Chen et al., 2007). Meanwhile, CRTC1, as another essential gene for long-term synaptic plasticity and synaptic development, also plays an important role in CREB-dependent gene transcription (Xue et al., 2015).

In the present study, we, for the first time, showed that DISS and TFSA possessed additive or synergistic protective effects on nerve regeneration in vitro. Additive effects were observed on cell viability, pCREB/CREB, BDNF, CRTC1, tNOS, and iNOS activities, synergistic effects were observed on BDNF mRNA. We adopted kinase inhibitors of U0126 and LY294002 to explore the mechanisms. It showed that combination of DISS and TFSA exerted an additive effect on CREB phosphorylation, and expressions of CRTC1 and BDNF at the protein level. Figure 6 illustrates that DISS and TFSA activated the PI$3 \mathrm{~K} /$ Akt and MAPK/Erk pathways, leading to stronger activation of the downstream CREB phosphorylation and simultaneously increased CRTC1 expression, and both of them were beneficial to the transcription of BDNF, which could eventually promote the neuronal survival.

In additional to the role in $\mathrm{CREB} / \mathrm{BDNF}$ regulation, $\mathrm{NO}$ is an important messenger and effector molecule that possesses neurotransmitter and neuromodulator functions and participates in physiological and pathological activities. NO, together with iNOS and nNOS, is well known to be involved in the pathogenesis of neurodegenerative disorders (Chen et al., 2007; Di et al., 2010; Lin et al., 2014). Both N-methyl-D-aspartate receptor activation and Glu can induce the iNOS expression and NO production, subsequently leading to excessive damage caused by free radicals (Akama and Van Eldik, 2000; Di et al., 2010). Our results indicated that the levels of iNOS and tNOS were significantly

\section{REFERENCES}

Akama, K. T., and Van Eldik, L. J. (2000). Beta-amyloid stimulation of inducible nitric-oxide synthase in astrocytes is interleukin-1beta- and tumor necrosis factor-alpha (TNFalpha)-dependent, and involves a TNFalpha receptorassociated factor- and NFkappaB-inducing kinase-dependent signaling mechanism. J. Biol. Chem. 275, 7918-7924.

Blumenthal, J. A., Smith, P. J., and Hoffman, B. M. (2012). Is Exercise a Viable Treatment for Depression? ACSMs Health Fit. J. 16, 14-21.

Chang, H. M., and But, P. P. H. (1986). Pharmacology and Applications of Chinese Material Medica. Toh Tuck Link: Word Scientific Publishing.

Chen, L. N., Sun, J., Yang, X. D., Xiao, K., Lv, Y., Zhang, B. Y., et al. (2016). The brain NO levels and NOS activities ascended in the early and middle stages and descended in the terminal stage in scrapie-infected animal models. Mol. Neurobiol. doi: 10.1007/s12035-016-9755-z [Epub ahead of print].

Chen, M. J., Nguyen, T. V., Pike, C. J., and Russo-Neustadt, A. A. (2007). Norepinephrine induces BDNF and activates the PI-3K and MAPK cascades in embryonic hippocampal neurons. Cell. Signal. 19, 114-128. doi: 10.1016/j.cellsig.2006.05.028 increased in Glu-treated SY5Y cells, while combination of DISS and TFSA could ameliorate such Glu-induced NO production and iNOS overdosage, suggesting that the combination effect on neuroprotection was also involved in regulation of iNOS and tNOS activities.

\section{CONCLUSION}

Combination of DISS and TFSA activated CREB-mediated MAPK and PI-3K cascades and induced the BDNF expression, revealing the underlying molecular mechanism of their additive neuroprotective effects. Our findings suggested the potential of a novel candidate oligosaccharide ester for the development of natural supplements against depression. Both DISS and TFSA are active oligosaccharide ester components found in the root of Polygala tenuifolia Willd. Our previous study showed that DISS has anti-depressive activity in vivo ( $\mathrm{Hu}$ et al., 2010). The molecular weights of DISS and TFSA are 752 and $682 \mathrm{kDa}$, respectively. And both of them are fat-soluble. However, it remains unclear whether DISS, TFSA or its metabolites could pass the blood brain barrier. In our future study, we will further explore their additive or synergistic effects on mental disorder in animal models and investigate whether they can pass the blood brain barrier.

\section{AUTHOR CONTRIBUTIONS}

$\mathrm{YH}, \mathrm{XD}$ and $\mathrm{PL}$ contributed in study design, $\mathrm{YH}, \mathrm{XL}$ and RZ contributed in trial and writing. DW contributed in writing.

\section{ACKNOWLEDGMENTS}

This work was supported by the National Natural Science Foundation of China for financial support of this study (No. 81573876).

Chou, T. C. (2006). Theoretical basis, experimental design, and computerized simulation of synergism and antagonism in drug combination studies. Pharmacol. Rev. 58, 621-681. doi: 10.1124/pr.58.3.10

Di, X., Yan, J., Zhao, Y., Zhang, J., Shi, Z., Chang, Y., et al. (2010). L-theanine protects the APP (Swedish mutation) transgenic SH-SY5Y cell against glutamate-induced excitotoxicity via inhibition of the NMDA receptor pathway. Neuroscience 168, 778-786. doi: 10.1016/j.neuroscience.2010.04.019

Dong, X. Z., Huang, C. L., Yu, B. Y., Hu, Y., Mu, L. H., and Liu, P. (2014). Effect of Tenuifoliside A isolated from Polygala tenuifolia on the ERK and PI3K pathways in C6 glioma cells. Phytomedicine 21, 1178-1188. doi: 10.1016/j.phymed.2014.04.022

Ghosh, A., Ginty, D. D., Bading, H., and Greenberg, M. E. (1994). Calcium regulation of gene expression in neuronal cells. J. Neurobiol. 25, 294-303. doi: $10.1002 /$ neu.480250309

Ginty, D. D., Bonni, A., and Greenberg, M. E. (1994). Nerve growth factor activates a Ras-dependent protein kinase that stimulates c-fos transcription via phosphorylation of CREB. Cell 77, 713-725. doi: 10.1016/0092-8674(94) 90055-8

Hardingham, G. E., Chawla, S., Cruzalegui, F. H., and Bading, H. (1999). Control of recruitment and transcription-activating function of CBP determines gene 
regulation by NMDA receptors and L-type calcium channels. Neuron 22, 789-798. doi: 10.1016/S0896-6273(00)80737-0

Hu, Y., Li, J., Liu, P., Chen, X., Guo, D. H., Li, Q. S., et al. (2012). Protection of SH-SY5Y neuronal cells from glutamate-induced apoptosis by 3,6'-disinapoyl sucrose, a bioactive compound isolated from Radix Polygala. J. Biomed. Biotechnol. 2012, 1-5. doi: 10.1155/2012/728342

Hu, Y., Liao, H. B., Dai-Hong, G., Liu, P., Wang, Y. Y., and Rahman, K. (2010). Antidepressant-like effects of 3,6'-disinapoyl sucrose on hippocampal neuronal plasticity and neurotrophic signal pathway in chronically mild stressed rats. Neurochem. Int. 56, 461-465. doi: 10.1016/j.neuint.2009.12.004

Hu, Y., Liao, H. B., Liu, P., Guo, D. H., and Rahman, K. (2009). A bioactive compound from Polygala tenuifolia regulates efficiency of chronic stress on hypothalamic-pituitary-adrenal axis. Pharmazie 64, 605-608.

Hu, Y., Liu, M., Liu, P., Guo, D. H., Wei, R. B., and Rahman, K. (2011). Possible mechanism of the antidepressant effect of 3,6'-disinapoyl sucrose from Polygala tenuifolia Willd. J. Pharm. Pharmacol. 63, 869-874. doi: 10.1111/j.20427158.2011.01281.X

Hu, Y., Liu, M. Y., Liu, P., Dong, X., and Boran, A. D. (2014). Neuroprotective effects of 3, 6'-disinapoyl sucrose through increased BDNF levels and CREB phosphorylation via the CaMKII and ERK1/2 pathway. J. Mol. Neurosci. 53, 600-607. doi: 10.1007/s12031-013-0226-y

Li, X. Y., Zhan, X. R., Liu, X. M., and Wang, X. C. (2011). CREB is a regulatory target for the protein kinase $\mathrm{Akt} / \mathrm{PKB}$ in the differentiation of pancreatic ductal cells into islet beta-cells mediated by hepatocyte growth factor. Biochem. Biophys. Res. Commun. 404, 711-716. doi: 10.1016/j.bbrc.2010.12.048

Lin, S. E., Wu, F. L., Wei, M. F., and Shen, L. J. (2014). Depletion of arginine by recombinant arginine deiminase induces nNOS-activated neurotoxicity in neuroblastoma cells. Biomed. Res. Int. 2014:589424. doi: 10.1155/2014/589424

Liu, P., Hu, Y., Guo, D. H., Wang, D. X., Tu, H. H., Ma, L., et al. (2010). Potential antidepressant properties of Radix Polygalae (Yuan Zhi). Phytomedicine 17, 794-799. doi: 10.1016/j.phymed.2010.01.004

Ma, X. H., Zheng, C. J., Han, L. Y., Xie, B., Jia, J., Cao, Z. W., et al. (2009). Synergistic therapeutic actions of herbal ingredients and their mechanisms from molecular interaction and network perspectives. Drug Discov. Today 14, 579-588. doi: 10.1016/j.drudis.2009.03.012

Majumder, S., Varadharaj, S., Ghoshal, K., Monani, U., Burghes, A. H., and Jacob, S. T. (2004). Identification of a novel cyclic AMP-response element
(CRE-II) and the role of CREB-1 in the cAMP-induced expression of the survival motor neuron (SMN) gene. J. Biol. Chem. 279, 14803-14811. doi: 10.1074/jbc.M308225200

Mathers, C., Fat, D., and Boerma, J. (2008). The Global Burden of Disease: 2004 Update. Geneva: World Health Organization.

Nair, A., and Vaidya, V. A. (2006). Cyclic AMP response element binding protein and brain-derived neurotrophic factor: molecules that modulate our mood? J. Biosci. 31, 423-434. doi: 10.1007/BF02704114

Reichardt, L. F. (2006). Neurotrophin-regulated signalling pathways. Philos. Trans. R. Soc. Lond. B Biol. Sci. 361, 1545-1564. doi: 10.1098/rstb.2006.1894

Roscilli, G., De Vitis, C., Ferrara, F. F., Noto, A., Cherubini, E., Ricci, A., et al. (2016). Human lung adenocarcinoma cell cultures derived from malignant pleural effusions as model system to predict patients chemosensitivity. J. Transl. Med. 14:61. doi: 10.1186/s12967-016-0816-x

Sakamoto, K., Karelina, K., and Obrietan, K. (2011). CREB: a multifaceted regulator of neuronal plasticity and protection. J. Neurochem. 116, 1-9. doi: 10.1111/j.1471-4159.2010.07080.x

Tan, Y., Rouse, J., Zhang, A., Cariati, S., Cohen, P., and Comb, M. J. (1996). FGF and stress regulate CREB and ATF-1 via a pathway involving p38 MAP kinase and MAPKAP kinase-2. EMBO J. 15, 4629-4642.

Xue, Z. C., Wang, C., Wang, Q. W., and Zhang, J. F. (2015). CREB-regulated transcription coactivator 1: important roles in neurodegenerative disorders. Sheng Li Xue Bao 67, 155-162.

Zhu, T., Yao, Q., Wang, W., Yao, H., and Chao, J. (2016). iNOS induces vascular endothelial cell migration and apoptosis via autophagy in ischemia/reperfusion injury. Cell Physiol. Biochem. 38, 1575-1588. doi: 10.1159/000443098

Conflict of Interest Statement: The authors declare that the research was conducted in the absence of any commercial or financial relationships that could be construed as a potential conflict of interest.

Copyright (c) 2016 Liu, Wang, Zhao, Dong, Hu and Liu. This is an open-access article distributed under the terms of the Creative Commons Attribution License (CC BY). The use, distribution or reproduction in other forums is permitted, provided the original author(s) or licensor are credited and that the original publication in this journal is cited, in accordance with accepted academic practice. No use, distribution or reproduction is permitted which does not comply with these terms. 